\title{
On a new unitarization scheme inspired by Dalitz and Tuan applied to meson-meson and meson-baryon scattering
}

\author{
Frieder Kleefeld ${ }^{* \dagger}$ \\ Centro de Física das Interacções Fundamentais (CFIF), \\ Instituto Superior Técnico, \\ Av. Rovisco Pais, 1049-001 Lisboa, Portugal \\ E-mail: kleefeldlefif.ist.utl.pt
}

\begin{abstract}
A new crossing symmetric unitarization scheme conveniently applied to meson-meson and meson-baryon scattering amplitudes is shortly proposed which can be not only used by theoreticians to unitarize arbitrary theoretical reaction amplitudes resulting from phenomenological Lagrangeans for mesons and baryons, yet also by experimentalists to generate realistic unitary fitting formulae for meson-meson and meson-baryon scattering observables sharing on one hand all the features of the underlying theoretical amplitudes, on the other hand allowing direct comparison to these amplitudes. The new unitarization scheme has been inspired by the Dalitz and Tuan (DT) representation [1], the basic ansatz of which is that “... the phases caused by different sources add ...” (using the words of B.S. Zou, D.V. Bugg, Phys. Rev. D 50 (1994) 591 [2]).
\end{abstract}

International Europhysics Conference on High Energy Physics

July 21st - 27th 2005

Lisboa, Portugal

\footnotetext{
*Speaker.

${ }^{\dagger}$ This work has been supported by the Fundação para a Ciência e a Tecnologia (FCT) of the Ministério da Ciência, Tecnologia e Ensino Superior of Portugal, under Grants no. PRAXIS XXI/BPD/20186/99, SFRH/BDP/9480/2002, POCTI/FNU/49555/2002, and POCTI/FP/FNU/50328/2003.
} 


\section{Unitarization and a new unitarization scheme inspired by Dalitz and Tuan}

A quantitative description of particle scattering/production processes involving strong interactions requires the complete non-perturbative scattering/production amplitude even close to threshold. We shall call the procedure of estimating the non-perturbative part of a scattering/production amplitude on the basis of a known perturbative or "tree-level" amplitude unitarization [3]. A comprehensive list of existing unitarization methods has been provided and discussed in Ref. [4]: the Padé method [5, 6], the Inverse Amplitude Method [4, 7, 8, 6], the N/D method, and K-matrix unitarization method [2, 包, 阳. Below we propose a to our best knowledge new crossing-symmetric unitarization method avoids drawbacks ${ }^{1}$ of above mentioned unitarization schemes and is inspired by the observation of Dalitz and Tuan (DT) [1] that — using the words of B.S. Zou and D.V. Bugg [2] - “... the phases caused by different sources add ...". ${ }^{2}$ Hence, we assume the S-matrix $S$ to be factorizable as a product of several partial S-matrices $S_{1}, \ldots, S_{n}(n \in \mathbb{N})$, i.e. $S=S_{1} S_{2} \ldots S_{n}$ $(n \in \mathbb{N})$. For simplicity we could assume unitarity of the partial S-matrices yielding the relation $S_{j}=1+2 i \bar{T}_{j}(j=1, \ldots, n)$ between partial S-matrices and corresponding partial T-matrices $\bar{T}_{1}, \ldots, \bar{T}_{n}$. Then the T-matrix could be expanded into partial T-matrices $\bar{T}_{1}, \ldots, \bar{T}_{n}$ as follows:

$$
T=\frac{(S-1)}{2 i}=\frac{1}{2 i}\left(\prod_{j=1}^{n} S_{j}-1\right)=\underbrace{\sum_{j=1}^{n} \bar{T}_{j}}_{\text {"tree-level" }}+\underbrace{\frac{1}{2 i}\left(\prod_{j=1}^{n}\left(1+2 i \bar{T}_{j}\right)-1\right)-\sum_{j=1}^{n} \bar{T}_{j}}_{\text {"unitarization correction" }} .
$$

An instructive case occurs when all $n$ partial T-matrices are equal to one partial T-matrix $\bar{T} \equiv$ $\bar{T}_{1}=\bar{T}_{2}=\ldots=\bar{T}_{n}$ corresponding to an unitary partial S-matrix $1+2 i \bar{T}$. Then we have $S=$ $(1+2 i \bar{T})^{n}$ and $T=(S-1) /(2 i)=\left((1+2 i \bar{T})^{n}-1\right) /(2 i)=n \bar{T}+$ "unitarization correction". Hence the $n$-th power of a partial S-matrix $1+2 i \bar{T}$ yields a "tree-level" T-matrix $n \bar{T}$ being $n$ times the corresponding partial T-matrix $\bar{T} .{ }^{3}$ Let's proceed to the case, in which we don't know, whether a

\footnotetext{
${ }^{1}$ Serious drawbacks of commonly used unitarization methods are lack of crossing symmetry (e.g. [10]), truncation dependences [8], problems with inclusion of chiral zeros [8], and difficulties to relate analytic expressions resulting from such methods directly to results obtained by quantum-field theoretic calculations of scattering amplitudes on the basis of Lagrangeans. Roy-equations [1] admit fortunately crossing symmetry by imposing external conditions, unfortunately they make strong assumptions about analyticity and have to be truncated in order to allow a numerical solution.

${ }^{2}$ Starting point for our consideration has been in analogy to DT the observation that the S-matrix for scalar isoscalar $\pi \pi \rightarrow \pi \pi$-scattering at energies below the $K \bar{K}$-threshold and slightly above the $\pi \pi$-threshold is fitted on the basis of experimental pole positions $M_{\sigma(600)}=(0.525-i 0.265) \mathrm{GeV}$ and $M_{f_{0}(980)}=(0.999-i 0.017) \mathrm{GeV}$ to an astonishing good approximation by a product of partial (Breit-Wigner) S-matrices $S_{\sigma(600)}(s)=\left(s-M_{\sigma(600)}^{* 2}\right) /\left(s-M_{\sigma(600)}^{2}\right)$ and $S_{f_{0}(980)}(s)=\left(s-M_{f_{0}(980)}^{* 2}\right) /\left(s-M_{f_{0}(980)}^{2}\right)$ and a background phase $S_{\sigma(600)}^{*}\left(s_{t h r}\right) S_{f_{0}(980)}^{*}\left(s_{t h r}\right)=\exp \left(2 i\left(-67.5^{\circ}\right)\right)$ being suitably chosen to make the cross section vanish exactly at the $\pi \pi$-threshold $s_{t h r}=4 m_{\pi^{ \pm}}^{2}$ (with $m_{\pi^{ \pm}}=0.13957018 \mathrm{GeV}$ ). The fit $S(s)=\exp \left(2 i\left(-67.5^{\circ}\right)\right) S_{\sigma(600)}(s) S_{f_{0}(980)}(s)$ - lacking by construction desirable square-root behaviour at $s=$ $s_{t h r}$ - is compared to experimental phaseshift data in Figs. 1, 2 and B. $S_{\sigma(600)}(s)$ and $S_{f_{0}(980)}(s)$ represent manifestly schannel $\pi \pi$-scattering, while the background phase $-67.5^{\circ}$ carries the reminder of $t$ - and $u$-channel scattering processes.

${ }^{3}$ It is straight forward to analytically continue this result to arbitrary rational values of $n$. For $n \in \mathbb{R} \backslash N_{0}$ we obtain:

$$
T=\frac{(1+2 i \bar{T})^{n}-1}{2 i}=n \bar{T} \text { (“tree-level”) }+\frac{1}{2 i} \sum_{j=2}^{\infty} \frac{n(n-1) \cdots(n-j+1)}{j !}(2 i \bar{T})^{j} \text { ("unitarization corr."). }
$$

A simple non-trivial example for a non-natural value of $n$ is $n=-1$, displaying a strong similarity to K-matrix unitarization, as $S=(1+2 i \bar{T})^{-1}$ and therefore $T=(S-1) /(2 i)=-\bar{T} /(1+2 i \bar{T})$.
} 
partial T-matrix $\bar{T}_{j}(j \in \mathbb{N})$ corresponds to an unitary partial S-matrix $S_{j}(j \in \mathbb{N})$ or not. Inspired by DT we would at least expect that the phase of $\bar{T}_{j}$ determines the phase of $S_{j}$ according to $S_{j}=$ $\left(\bar{T}_{j} / \bar{T}_{j}^{*}\right)^{\alpha_{j}} \equiv\left(1+2 i \operatorname{Im}\left[\bar{T}_{j}\right] \bar{T}_{j}^{*-1}\right)^{\alpha_{j}}$, while $\alpha_{j}$ is determined such that the "tree-level" contribution to the T-matrix is given by $\bar{T}_{j}$ itself. To find $\alpha_{j}$ we expand $\left(S_{j}-1\right) /(2 i)$ in terms of $\bar{T}_{j}$ :

$$
\frac{S_{j}-1}{2 i}=\underbrace{\alpha_{j} \frac{\operatorname{Im}\left[\bar{T}_{j}\right]}{\bar{T}_{j}^{*}}}_{\text {"tree-level" }}+\underbrace{\frac{1}{2 i} \sum_{\ell=2}^{\infty} \frac{1}{\ell !}\left(2 i \alpha_{j} \frac{\operatorname{Im}\left[\bar{T}_{j}\right]}{\bar{T}_{j}^{*}}\right)^{\ell} \prod_{k=1}^{\ell}\left(1-\frac{k-1}{\alpha_{j}}\right)}_{\text {"unitarization correction" }} .
$$

Simple inspection yields $\alpha_{j} \operatorname{Im}\left[\bar{T}_{j}\right] / \bar{T}_{j}^{*}=\bar{T}_{j} \Rightarrow \alpha_{j}=\left|\bar{T}_{j}\right|^{2} / \operatorname{Im}\left[\bar{T}_{j}\right] .{ }^{4}$ Hence we conclude: If the "tree-level" T-matrix is given by a sum $\bar{T}_{1}+\ldots+\bar{T}_{n}(n \in \mathbb{N})$ of arbitrary partial T-matrices $\bar{T}_{1}, \ldots$, $\bar{T}_{n}$, then a DT-unitarized S-matrix $S$ with a "tree-level" term $\bar{T}_{1}+\ldots+\bar{T}_{n}$ can be denoted as:

$$
\begin{aligned}
& S=S_{1} S_{2} \ldots S_{n}=\left(\frac{\bar{T}_{1}}{\bar{T}_{1}^{*}}\right)^{\frac{\left|\bar{T}_{1}\right|^{2}}{\operatorname{Im}\left[\bar{T}_{1}\right]}}\left(\frac{\bar{T}_{2}}{\bar{T}_{2}^{*}}\right)^{\frac{\left|\bar{T}_{2}\right|^{2}}{\operatorname{Im}\left[\bar{T}_{2}\right]}} \ldots\left(\frac{\bar{T}_{n}}{\bar{T}_{n}^{*}}\right)^{\frac{\left|\bar{T}_{n}\right|^{2}}{\operatorname{Im}\left[\bar{T}_{n}\right]}}= \\
& =\exp \left[i\left(\frac{\left|\bar{T}_{1}\right|^{2}}{\operatorname{Im}\left[\bar{T}_{1}\right]} \arg \left(\frac{\bar{T}_{1}}{\bar{T}_{1}^{*}}\right)+\frac{\left|\bar{T}_{2}\right|^{2}}{\operatorname{Im}\left[\bar{T}_{2}\right]} \arg \left(\frac{\bar{T}_{2}}{\bar{T}_{2}^{*}}\right)+\ldots+\frac{\left|\bar{T}_{n}\right|^{2}}{\operatorname{Im}\left[\bar{T}_{n}\right]} \arg \left(\frac{\bar{T}_{n}}{\bar{T}_{n}^{*}}\right)\right)\right] \text {. }
\end{aligned}
$$

\section{Instructive examples}

DT-unitarized s-channel Breit-Wigner resonance. Consider a partial T-matrix for one schannel Breit-Wigner resonance with constant complex mass $M$ dressed by a real coupling constant $g \in \mathbb{R}$. I.e. we make the ansatz $\bar{T}_{1}=g \operatorname{Im}\left[M^{2}\right] /\left(s-M^{2}\right)$ yielding $\operatorname{Im}\left[\bar{T}_{1}\right]=g \operatorname{Im}^{2}\left[M^{2}\right] /\left|s-M^{2}\right|^{2}$ and $\left|\bar{T}_{1}\right|^{2}=g^{2} \operatorname{Im}^{2}\left[M^{2}\right] /\left|s-M^{2}\right|^{2}$. Then the unitarized S-matrix inspired by DT will be given by $S=\left(\frac{s-M^{* 2}}{s-M^{2}}\right)^{g}=\exp \left[i g \arg \left(\frac{s-M^{* 2}}{s-M^{2}}\right)\right]$. The resulting DT-unitarized T-matrix is given by:

$$
T=\frac{S-1}{2 i}=\underbrace{g \frac{\operatorname{Im}\left[M^{2}\right]}{\left(s-M^{2}\right)}}_{\text {"tree-level" }}+\underbrace{\frac{1}{2 i} \sum_{\ell=2}^{\infty} \frac{g(g-1)(g-2) \cdots(g-\ell+1)}{\ell !}\left(2 i \frac{\operatorname{Im}\left[M^{2}\right]}{\left(s-M^{2}\right)}\right)^{\ell}}_{\text {"unitarization correction" }} .
$$

In agreement with our previous discussion the unitarized T-matrix $T$ reduces to the "tree-level" T-matrix $T_{1}$ for $g \in \mathbb{N}_{0}$. $T$ seems to possess the same poles and zeros as $T_{1}$.

DT-unitarized one-channel two-resonance $L \sigma M$ approach to $\pi \pi$-scattering. The "treelevel" $U(3) \times U(3)$ Linear Sigma Model $(\mathrm{L} \sigma \mathrm{M})$ scattering amplitude for $\pi \pi \rightarrow \pi \pi$ scattering with $\sigma(600)$ and $f_{0}(980)$ intermediate states is given by $\left(f_{\pi} \simeq 92.42 \mathrm{MeV}\right):{ }^{5}$

$$
\begin{aligned}
& \bar{T}_{\pi^{0} \pi^{0} \leftarrow \pi^{+} \pi^{-}}(s, t, u)=\sqrt{\rho_{\pi \pi}(s)} \frac{s-m_{\pi}^{2}}{f_{\pi}^{2}}\left(1-\left(s-m_{\pi}^{2}\right)\left(\frac{\cos ^{2} \phi_{s}}{s-M_{\sigma}^{2}}+\frac{\sin ^{2} \phi_{s}}{s-M_{f_{0}}^{2}}\right)\right) \sqrt{\rho_{\pi \pi}(s)}= \\
& =\sqrt{\rho_{\pi \pi}(s)}\left(\frac{s-m_{\pi}^{2}}{f_{\pi}^{2}}-\frac{\left(s-m_{\pi}^{2}\right)^{2}}{f_{\pi}^{2} \operatorname{Im}\left[M_{\sigma}^{2}\right]} \cos ^{2} \phi_{s} \frac{\operatorname{Im}\left[M_{\sigma}^{2}\right]}{s-M_{\sigma}^{2}}-\frac{\left(s-m_{\pi}^{2}\right)^{2}}{f_{\pi}^{2} \operatorname{Im}\left[M_{f_{0}}^{2}\right]} \sin ^{2} \phi_{s} \frac{\operatorname{Im}\left[M_{f_{0}}^{2}\right]}{s-M_{f_{0}}^{2}}\right) \sqrt{\rho_{\pi \pi}(s)} .
\end{aligned}
$$

${ }^{4}$ The choice implies of course $\frac{S_{j}-1}{2 i}=\bar{T}_{j}$ ("tree-level”) $+\frac{1}{2 i} \sum_{\ell=2}^{\infty} \frac{\left(2 i \bar{T}_{j}\right)^{\ell}}{\ell !} \prod_{k=1}^{\ell}\left(1-(k-1) \frac{\operatorname{Im}\left[\bar{T}_{j}\right]}{\left|\bar{T}_{j}\right|^{2}}\right)$ ("unit. corr.").

${ }^{5} \phi_{s} \in \mathbb{R}$ is a nonstrange-strange scalar mixing angle defined by $|\sigma\rangle=\cos \phi_{s}|n \bar{n}\rangle-\sin \phi_{s}|s \bar{s}\rangle$ and $\left|f_{0}\right\rangle=\sin \phi_{s}|n \bar{n}\rangle+$ $\cos \phi_{s}|s \vec{s}\rangle$, while $\rho_{\pi \pi}(s)=\left|\vec{p}_{c m}^{\pi \pi}\right| /(8 \pi \sqrt{s})=\sqrt{\left(1-4 m_{\pi}^{2} s^{-1}\right)} /(16 \pi) \simeq \theta\left(s-4 m_{\pi}^{2}\right) /(16 \pi)$ is the $\pi \pi$-phasespace. 

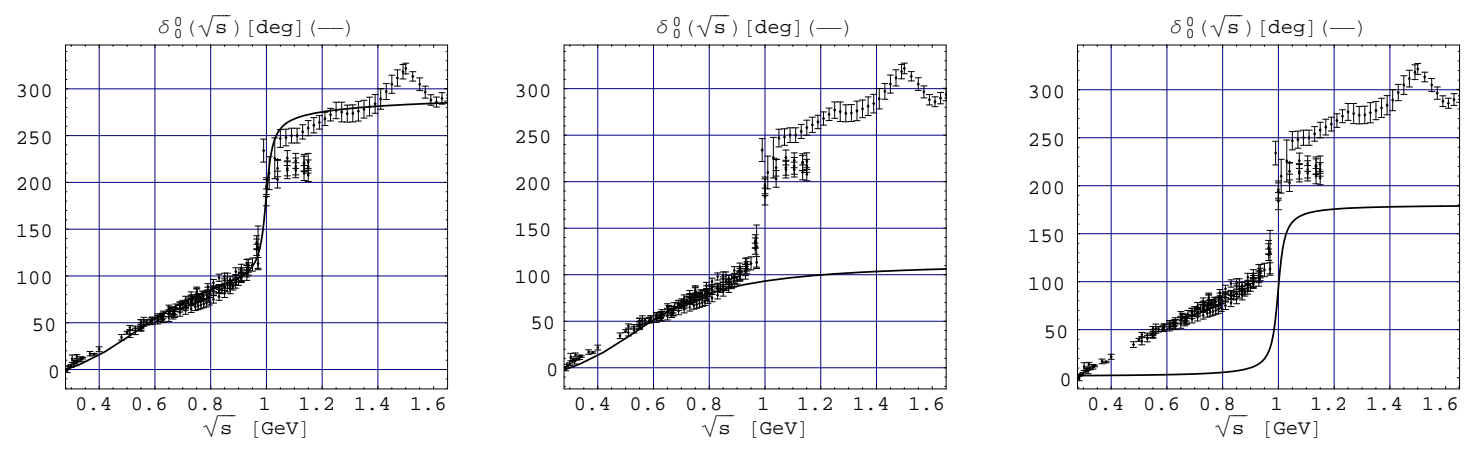

Figure 1: Fit of $\delta_{0}^{0}(\sqrt{s})$ : contri- Figure 2: Fit of $\delta_{0}^{0}(\sqrt{s})$ : contribution of "background" $\left(-67.5^{\circ}\right)$ bution of "background" $\left(-67.5^{\circ}\right)$

Figure 3: Fit of $\delta_{0}^{0}(\sqrt{s})$ : contribuand $\sigma(600)$ and $f_{0}(980)$. and $\sigma(600)$.

The DT-unitarized crossing symmetric $S$-matrix is now obviously $\left(P_{I=0,1,2}\right.$ are isospin projectors)

$$
\begin{aligned}
& S_{\pi \pi \leftarrow \pi \pi}(s, t, u)=\exp \left[i \sqrt{\rho_{\pi \pi}(s)} \times\right. \\
& \left.\quad \times\left(3 P_{I=0}(\Delta(s)+\Delta(t)+\Delta(u))+P_{I=1}(\Delta(t)-\Delta(u))+P_{I=2}(\Delta(t)+\Delta(u))\right) \sqrt{\rho_{\pi \pi}(s)}\right]
\end{aligned}
$$

with $\Delta(s) \equiv-\frac{\left(s-m_{\pi}^{2}\right)^{2}}{f_{\pi}^{2} \operatorname{Im}\left[M_{\sigma}^{2}\right]} \cos ^{2} \phi_{s} \arg \left(\frac{s-M_{\sigma}^{* 2}}{s-M_{\sigma}^{2}}\right)-\frac{\left(s-m_{\pi}^{2}\right)^{2}}{f_{\pi}^{2} \operatorname{Im}\left[M_{f_{0}}^{2}\right]} \sin ^{2} \phi_{s} \arg \left(\frac{s-M_{f_{0}}^{* 2}}{s-M_{f_{0}}^{2}}\right)$.

\section{References}

[1] R.H. Dalitz, S. Tuan, Annals Phys. (N.Y.) 10 (1960) 307; B.S. Zou et al., Phys. Rev. D 48 (1993) 3948.

[2] B.S. Zou, D.V. Bugg, Phys. Rev. D 50 (1994) 591.

[3] F. Kleefeld, nucl-th/0510017 ；F. Kleefeld, AIP Conf. Proc. 717 (2004) 332.

[4] T.N. Truong, Phys. Rev. Lett. 67 (1991) 2260.

[5] J.L. Basdevant, Fortsch. Phys. 20 (1972) 283; J.L. Basdevant, B.W. Lee, Phys. Lett. B 29 (1969) 437; S. Willenbrock, Phys. Rev. D 43 (1991) 1710; D.A. Dicus, W.W. Repko, Phys. Rev. D 42 (1990) 3660.

[6] T.N. Truong, Phys. Rev. Lett. 61 (1988) 2526; A. Dobado, M.J. Herrero, T.N. Truong, Phys. Lett. B 235 (1990) 129, 235 (1990) 134.

[7] E.P. Tryon, Phys. Rev. D 4 (1971) 1202; A. Dobado, J.R. Peláez, Phys. Rev. D 47 (1993) 4883; T. Hannah, Phys. Rev. D 51 (1995) 103, 52 (1995) 4971, 55 (1997) 5613; J.R. Peláez, A. Gómez Nicola, Nucl. Phys. A 675 (2000) 96C; J. Nieves et al., Phys. Rev. D 65 (2002) 036002.

[8] A. Dobado, J.R. Peláez, Phys. Rev. D 56 (1997) 3057; T. Hannah, Phys. Rev. D 59 (1999) 057502.

[9] S.U. Chung et al., Annalen Phys. (Leipzig) 4 (1995) 404; D. Black, A. H. Fariborz, S. Moussa, S. Nasri, J. Schechter, Phys. Rev. D 64 (2001) 014031.

[10] I.P. Cavalcante, J. Sa Borges, AIP Conf. Proc. 631 (2003) 713.

[11] S.M. Roy, Phys. Lett. B 36 (1971) 353; B. Ananthanarayan, G. Colangelo, J. Gasser, H. Leutwyler, Phys. Rept. 353 (2001) 207; G. Mahoux, Roy Type Equations For Pion Pion Partial Waves With Exact Crossing Symmetry Builtin. (Talk), in Formigal 1974, Proc. 2nd Int. Winter Meeting on Fundamental Physics, Madrid 1974, 267-280; D. Atkinson, T.P. Pool, Nucl. Phys. B 81 (1974) 502. 